Case Report

\title{
Myelodysplastic Syndrome/Acute Myeloid Leukemia Arising in Idiopathic Erythrocytosis
}

\author{
Stephen E. Langabeer ${ }^{(D)}{ }^{1}$ Eibhlin Conneally, ${ }^{2}$ and Catherine M. Flynn ${ }^{2}$ \\ ${ }^{1}$ Cancer Molecular Diagnostics, St. James's Hospital, Dublin 8, Ireland \\ ${ }^{2}$ Department of Haematology, St. James's Hospital, Dublin 8, Ireland \\ Correspondence should be addressed to Stephen E. Langabeer; slangabeer@stjames.ie
}

Received 27 November 2017; Accepted 7 February 2018; Published 22 February 2018

Academic Editor: Sergio Storti

Copyright ( $\odot 2018$ Stephen E. Langabeer et al. This is an open access article distributed under the Creative Commons Attribution License, which permits unrestricted use, distribution, and reproduction in any medium, provided the original work is properly cited.

The term "idiopathic erythrocytosis (IE)" is applied to those cases where a causal clinical or pathological event cannot be elucidated and likely reflects a spectrum of underlying medical and molecular abnormalities. The clinical course of a patient with IE is described manifesting as a persistent erythrocytosis with a low serum erythropoietin level, mild eosinophilia, and with evidence of a thrombotic event. The patient subsequently developed a myelodysplasic syndrome (MDS) and acute myeloid leukemia (AML), an event not observed in erythrocytosis patients other than those with polycythemia vera (PV). Application of a next-generation sequencing (NGS) approach targeted for myeloid malignancies confirmed wild-type JAK2 exons 12-15 and identified a common SH2B3 W262R single-nucleotide polymorphism associated with the development of hematological features of myeloproliferative neoplasms (MPNs). Further NGS analysis detected a CBL L380P mutated clone expanding in parallel with the development of MDS and subsequent AML. Despite the absence of JAK2, MPL exon 10, or CALR exon 9 mutations, a similarity with the disease course of PV/MPN was evident. A clonal link between the erythrocytosis and AML could be neither confirmed nor excluded. Future molecular identification of the mechanisms underlying IE is likely to provide a more refined therapeutic approach.

\section{Introduction}

The causes of erythrocytosis are many and can be broadly divided into either primary or secondary forms. Primary causes are due to a defect intrinsic to the erythroid compartment of the bone marrow which leads to increased red cell generation, whereas secondary causes are due to factors external to the bone marrow that are produced in excess and drive red cell production [1]. The most common cause of acquired primary erythrocytosis is the myeloproliferative neoplasm (MPN) of polycythemia vera (PV) that is molecularly characterized by the JAK2 p.V617F and exon 12 mutations [2]. Mutations of other genes in the erythropoiesis, oxygen sensing, and oxygen transport pathways are known to result in erythrocytosis; however, the underlying causes are unknown in a large number of cases, particularly those recognized as congenital erythrocytosis, and remain classified as idiopathic erythrocytosis (IE) [3]. Several clinical and biological differences exist between IE and PV, including a considerably lower risk of thrombotic events in IE patients compared to PV patients [4] with transformation to acute myeloid leukemia (AML) exceedingly rare in individuals with IE or other molecularly annotated forms of erythrocytosis [5]. A case is described in which application of the myeloid malignancy-targeted, next-generation sequencing (NGS) approach retrospectively provided insights into the molecular appearance of myelodysplastic syndrome (MDS)/AML in a patient with IE.

\section{Case Report}

An overweight 62-year-old male with hypertension and hyperlipidemia presented with a hemoglobin level of $20.4 \mathrm{~g} / \mathrm{dL}$, hematocrit of 0.59 , normal white cell and platelet counts, and a mild eosinophilia (Table 1). The patient had no clinical signs of PV, normal spleen size, normal oxygen 
TABLE 1: Hematological indices and molecular analysis throughout the patient's clinical course.

\begin{tabular}{|c|c|c|c|c|c|c|c|c|c|}
\hline $\begin{array}{l}\text { Time } \\
\text { (months) }\end{array}$ & $\mathrm{Hb}$ & HCT & RCC & PLT & WCC & Eos & $\begin{array}{c}\text { BM blasts } \\
\text { (IP/morphology) }\end{array}$ & $\begin{array}{l}\text { Mutations detected (variant allele } \\
\text { frequency) }\end{array}$ & \\
\hline $\mathrm{Dx}$ & 20.4 & 0.59 & 6.20 & 140 & 5.8 & 1.0 & - & - & \multirow{5}{*}{$\begin{array}{c}C B L \text { p.L380P } \\
(3.6 \%)\end{array}$} \\
\hline 41 & 16.2 & 0.49 & 6.29 & 158 & 6.8 & 0.9 & - & SH2B3 p.W262R (52.4\%) & \\
\hline 149 & 19.7 & 0.58 & 6.78 & 75 & 8.1 & 1.9 & - & SH2B3 p.W262R (45.9\%) & \\
\hline 157 & 8.9 & 0.26 & 2.62 & 112 & 4.0 & 0.2 & $4 \% / 0 \%$ & - & \\
\hline 159 & 10.1 & 0.30 & 3.00 & 106 & 3.3 & 0.1 & $8 \% / 6 \%$ & - & \\
\hline 161 & 10.1 & 0.29 & 3.19 & 35 & 6.1 & 0.4 & $15 \% / 22 \%$ & SH2B3 p.W262R (56.8\%) & $\begin{array}{c}C B L \text { p.L380P } \\
(37.2 \%)\end{array}$ \\
\hline
\end{tabular}

$\mathrm{Hb}$ : hemoglobin (g/dL); HCT: hematocrit; RCC: red cell count $\left(\times 10^{12} / \mathrm{L}\right)$; PLT: platelet count $\left(\times 10^{9} / \mathrm{L}\right)$; WCC: white cell count $\left(\times 10^{9} / \mathrm{L}\right)$; Eos: eosinophil count $\left(\times 10^{9} / \mathrm{L}\right)$; BM blasts: bone marrow myeloblasts; IP: immunophenotyping; Dx: diagnosis.

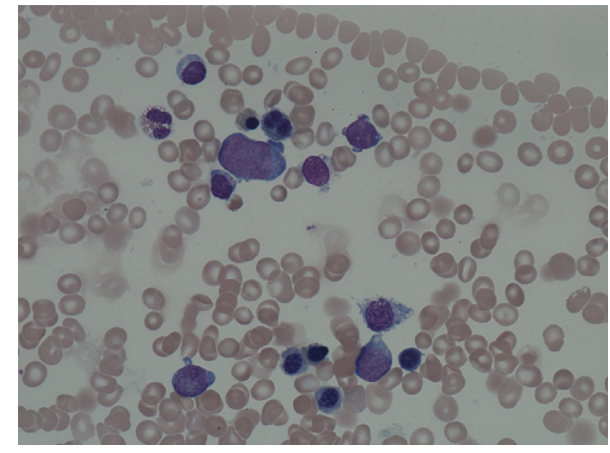

FIGURE 1: Bone marrow morphology at development of myelodysplasia/acute myeloid leukemia showing myeloblasts and dysplastic red blood cell precursors.

saturation, no endogenous erythrocyte colony formation, no evidence of abnormal hemoglobin, and no family history of a hematological abnormality. The patient was managed with intermittent venesection alone for nine years with no evidence of the JAK2 p.V617F mutations by allele-specific PCR or the JAK2 exon 12 mutations by high-resolution melt curve analysis, excluding a diagnosis of PV [2]. At 149 months after diagnosis, thrombocytopenia $\left(75 \times 10^{9} / \mathrm{L}\right)$ and increasing eosinophilia $\left(1.9 \times 10^{9} / \mathrm{L}\right)$ were noted with a low serum erythropoietin (EPO) level of $0.6 \mathrm{IU} / \mathrm{L}$ (normal range 2.6$18.5 \mathrm{IU} / \mathrm{L})$. At 154 months after diagnosis, the patient had temporoparietal stroke. Worsening pancytopenia at 157 months prompted bone marrow biopsy which demonstrated $4 \%$ myeloblasts (Table 1). Subsequent bone marrow investigations showed progressively increasing myeloblast cell numbers by both morphological and immunophenotypic evaluation with reduced dysplastic megakaryocytes, dyserythropoiesis with basophilic stippling, and binuclear red cell forms, all consistent with MDS progressing to AML (Figure 1). The karyotype at 159 months was normal. The patient was not fit for intensive treatment and was given best supportive care with $\mathrm{EPO}$, intermittent red cell transfusions, and low-dose steroids but died of infection at 161 months.

A targeted NGS approach panel was retrospectively employed to detect mutations possibly contributing to the erythrocytosis and subsequent development of MDS/AML in archival peripheral blood or bone marrow DNA samples from 41, 149, and 161 months. Amplicon libraries covering thirty commonly mutated genes implicated in myeloid malignancies, either covering the entire coding region (CALR, CEBPA, ETV6, EZH2, RUNX1, SH2B3, TET2, TP53, and ZRSR2) or mutational hotspots ( $A B L 1, A S X L 1, B R A F$, CBL, CSF3R, DNMT3A, FLT3, GATA2, IDH1, IDH2, JAK2, KIT, KRAS, MPL, NPM1, NRAS, PTPN11, SETBP1, SF3B1, $S R S F 2$, and $U 2 A F 1$, were generated. Sequencing was performed with Ion AmpliSeq ${ }^{\mathrm{TM}}$ methodology (Thermo Fisher Scientific, Paisley, UK). Calling of somatic mutations was achieved using an algorithm that excluded synonymous mutations, variants located within intronic or untranslated regions, and those present at a variant allele frequency (VAF) of $<5 \%$. A minimum target depth of coverage for variant calls was set at 500x as previously described [6, 7]. The nonsynonymous $\mathrm{SH} 2 \mathrm{~B} 3$ exon 2, single-nucleotide polymorphism (SNP) W262R (c.784T>C, NP_005466.1, rs3184504) was present at all three time points analyzed at VAFs of approximately 50\% (Table 1). No mutations were detected in $M P L$ exon 10 or CALR exon 9. A single $C B L$ L380P mutation (c.1139T $>C, N P \_005179.2$ ) was detected in the bone marrow sample at 161 months. Reinterrogation of sequencing data revealed this $C B L$ mutation to be present a year previously at a VAF of $3.6 \%$ (Table 1).

\section{Discussion}

Despite the absence of either the JAK2 V617F or exon 12 mutations in the patient, a high degree of suspicion remained throughout the clinical course for a diagnosis of PV or "PV-like" MPN given the persistently raised hematocrit, the low serum EPO, a mild eosinophilia, and clinically a thrombotic episode (stroke). While NGS confirmed the absence of the JAK2 V617F and exon 12 mutations, several alternative mutations of JAK2 have been identified in sporadic cases of "PV-like" MPN and hereditary erythrocytosis [8-13], yet none were identified by NGS in exons $12-15$ in the patient.

Of interest is the presence of the SH2B3 W262R SNP. $S H 2 B 3$ (formerly $L N K$ ) encodes the $L N K$ inhibitory adaptor protein that modulates thrombopoietin and erythropoietin signalling by interacting with JAK2 and inhibiting downstream STAT activation. Disruption of this function by a mutant protein results in aberrant JAK-STAT signalling and cytokine responsiveness. Low frequency but recurrent, acquired, and germ line mutations of $\mathrm{SH} 2 \mathrm{~B} 3$ have been 
reported in both sporadic and familial MPN, respectively, particularly in those cases and kindred identified with IE $[14,15]$. While no somatic mutations were identified within the entire coding region of $S H 2 B 3$, this patient was heterozygous (T/C) for the common W262R SNP [16]. This SNP and others within SH2B3 have been shown to be associated with an increase in platelets, eosinophils, and elevated hemoglobin and hematocrit levels [17-22]. Furthermore, some recent evidence exists for the T allele of this SNP to be associated with the development of MPN and JAK2 V617Fpositive hematopoiesis $[16,21,22]$. Despite in vitro functional analysis of another nonsynonymous SH2B3 SNP (E400K) in a patient with IE indicating no impairment of SH2B3 inhibiting JAK2-STAT5 activation, subtle loss of function induced by $S H 2 B 3$ SNPs cannot be excluded [23]. While tempting to speculate a causal role for this SH2B3 SNP in the development of erythrocytosis in this patient, no specific evidence exists for such an association, suggesting involvement of additional genetic and/or epigenetic events.

Transformation to AML is a recurrent event in PV with reported risks of $2.3-14.4 \%$ at ten years [24]. However, transformation to or development of MDS/AML in other forms of molecularly annotated erythrocytosis or IE is exceedingly rare [25]. A true transformation of the erythrocytosis could neither be confirmed nor excluded in this case due to the absence of a pre-MDS/AML marker of clonality with the possibility that the erythrocytosis and MDS/AML represent two unrelated pathologies. Activating mutations of $C B L$, a negative regulator of receptor tyrosine kinases, including the p.L380P detected in this case, is recurrent in myeloid malignancies and is associated with progression of MDS to AML [26].

In conclusion, we describe a patient with IE possessing a clinical similarity to PV in which there are persistent erythrocytosis, a thrombotic event, and the acquisition of somatic mutations that resulted in MDS/AML. Employment of an NGS gene panel specifically targeted for investigation of IE has recently demonstrated the benefits of this type of approach [27]. The potential exists for identifying those patients at increased risk of developing a myeloid malignancy, enabling refined counseling and therapeutic decision-making throughout the disease course.

\section{Conflicts of Interest}

The authors declare that there are no conflicts of interest regarding the publication of this paper.

\section{References}

[1] G. Lee and M. O. Arcasoy, "The clinical and laboratory evaluation of the patient with erythrocytosis," European Journal of Internal Medicine, vol. 26, no. 5, pp. 297-302, 2015.

[2] D. A. Arber, A. Orazi, R. Hasserjian et al., "The 2016 revision to the World Health Organization classification of myeloid neoplasms and acute leukemia," Blood, vol. 127, no. 20, pp. 2391-2405, 2016.

[3] C. Bento, H. Almeida, T. M. Maia et al., "Molecular study of congenital erythrocytosis in 70 unrelated patients revealed a potential causal mutation in less than half of the cases (where is/are the missing gene(s)?)," European Journal of Haematology, vol. 91, no. 4, pp. 361-368, 2013.

[4] I. Bertozzi, M. Ruggeri, I. Nichele, G. Biagetti, E. Cosi, and M. L. Randi, "Thrombotic and hemorrhagic complications in idiopathic erythrocytosis," American Journal of Hematology, vol. 92, no. 11, pp. E639-E641, 2017.

[5] M. F. McMullin, "Investigation and management of erythrocytosis," Current Hematologic Malignancy Reports, vol. 11, no. 5, pp. 342-347, 2016.

[6] K. Haslam, M. A. Catherwood, E. Dobbin, A. Sproul, S. E. Langabeer, and K. I. Mills, "Inter-laboratory evaluation of a next-generation sequencing panel for acute myeloid leukemia," Molecular Diagnosis \& Therapy, vol. 20, no. 5, pp. 457-461, 2016.

[7] S. E. Langabeer, K. Haslam, and C. M. Flynn, "Isolated erythrocytosis associated with a CALR mutation," Blood Cells, Molecules \& Diseases, vol. 66, pp. 6-7, 2017.

[8] S. Schnittger, U. Bacher, W. Kern, M. Schröder, T. Haferlach, and C. Schoch, "Report on two novel nucleotide exchanges in the JAK2 pseudo-kinase domain: D620E and E627E," Leukemia, vol. 20, no. 12, pp. 2195-2197, 2006.

[9] F. Grünebach, U. Bross-Bach, L. Kanz, and P. Brossart, "Detection of a new D620E mutation in addition to V617F in a patient with polycythemia vera," Leukemia, vol. 20, no. 12, pp. 2210-2211, 2006.

[10] B. Bahar, K. Barton, and A. R. Kini, "The role of exon 13 G571S JAK2 mutation in myeloproliferative neoplasms," Leukemia Research Reports, vol. 6, pp. 27-28, 2016.

[11] C. Cleyrat, J. Jelinek, F. Girodon et al., "JAK2 mutation and disease phenotype: a double L611/V617F in cis mutation of $J A K 2$ is associated with isolated erythrocytosis and increased activation of AKT and ERK1/2 rather than STAT5," Leukemia, vol. 34, no. 5, pp. 1069-1073, 2010.

[12] I. S. Tiong, D. A. Casolari, S. Moore et al., "Apparent JAK2-negative polycythaemia vera due to compound mutations in exon 14," British Journal of Haematology, vol. 178, no. 2, pp. 333-336, 2017.

[13] K. Kapralova, H. Horvathova, C. Pecquet et al., "Cooperation of germ line JAK2 mutations E845D and R1063H in hereditary erythrocytosis with megakaryocytic atypia," Blood, vol. 128, no. 10, pp. 1418-1423, 2016.

[14] M. F. McMullin and H. Cario, "LNK mutations and myeloproliferative disorders," American Journal of Hematology, vol. 91, no. 2, pp. 248-251, 2016.

[15] N. Maslah, B. Cassinat, E. Verger, J. J. Kiladjian, and L. Velazquez, "The role of $L N K / S H 2 B 3$ genetic alterations in myeloproliferative neoplasms and other hematological disorders," Leukemia, vol. 31, no. 8, pp. 1661-1670, 2017.

[16] E. Lesteven, M. Picque, C. Conejero Tonetti et al., "Association of a single-nucleotide polymorphism in the $\mathrm{SH} 2 \mathrm{~B} 3$ gene with JAK2 V617F-positive myeloproliferative neoplasms," Blood, vol. 123, no. 5, pp. 794-796, 2014.

[17] M. K. Christiansen, S. B. Larsen, M. Nyegaard et al., "The $\mathrm{SH} 2 \mathrm{~B} 3$ and KCNK5 loci may be implicated in regulation of platelet count, volume, and maturity," Thrombosis Research, vol. 158, pp. 86-92, 2017.

[18] D. F. Gudbjartsson, U. S. Bjomsdottir, E. Halapi et al., "Sequence variants affecting eosinophil numbers associate with asthma and myocardial infarction," Nature Genetics, vol. 41, no. 3, pp. 342-347, 2009.

[19] M. E. Daly, "Determinants of platelet count in humans," Haematologica, vol. 96, no. 1, pp. 10-13, 2011.

[20] S. K. Ganesh, N. A. Zakai, F. J. A. van Rooiij et al., "Multiple loci influence erythrocyte phenotypes in the CHARGE consortium," Nature Genetics, vol. 41, no. 11, pp. 1191-1198, 2009. 
[21] Y. Chen, F. Fang, Y. Hu et al., "The polymorphisms in LNK gene correlated to the clinical type of myeloproliferative neoplasms," PLoS One, vol. 11, no. 4, article e0154183, 2016.

[22] D. A. Hinds, K. E. Barnholt, R. A. Mesa et al., "Germ line variants predispose to both JAK2 V617F clonal hematopoiesis and myeloproliferative neoplasms," Blood, vol. 128, no. 8, pp. 1121-1128, 2016.

[23] M. F. McMullin, C. Wu, M. J. Percy, and W. Tong, "A nonsynonymous $L N K$ polymorphism associated with idiopathic erythrocytosis," American Journal of Hematology, vol. 86, no. 11, pp. 962-964, 2011.

[24] S. Cerquozzi and A. Tefferi, "Blast transformation and fibrotic progression in polycythemia vera and essential thrombocythemia: a literature review of incidence and risk factors," Blood Cancer Journal, vol. 5, no. 11, p. e366, 2015.

[25] K. Labno-Kirszniok, T. Niesporek, A. Wiecek, G. Helbig, and J. Lubinski, "Acute myeloid leukemia in a 38-year-old hemodialyzed patient with von Hippel-Lindau disease," Hereditary Cancer in Clinical Practice, vol. 11, no. 1, p. 11, 2013.

[26] C. Reindl, H. Quentmeier, K. Petropoulos et al., "CBL exon 8/9 mutants activate the FLT3 pathway and cluster in core binding factor/11q deletion acute myeloid leukemia/myelodysplastic syndrome subtypes," Clinical Cancer Research, vol. 15, no. 7, pp. 2238-2247, 2009.

[27] C. Camps, N. Petousi, C. Bento et al., "Gene panel sequencing improves the diagnostic work-up of patients with idiopathic erythrocytosis and identifies new mutations," Haematologica, vol. 101, no. 11, pp. 1306-1318, 2016. 


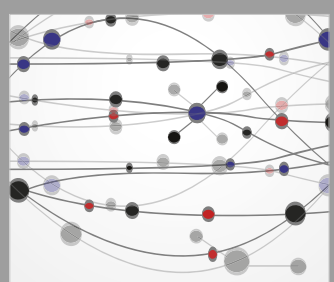

The Scientific World Journal
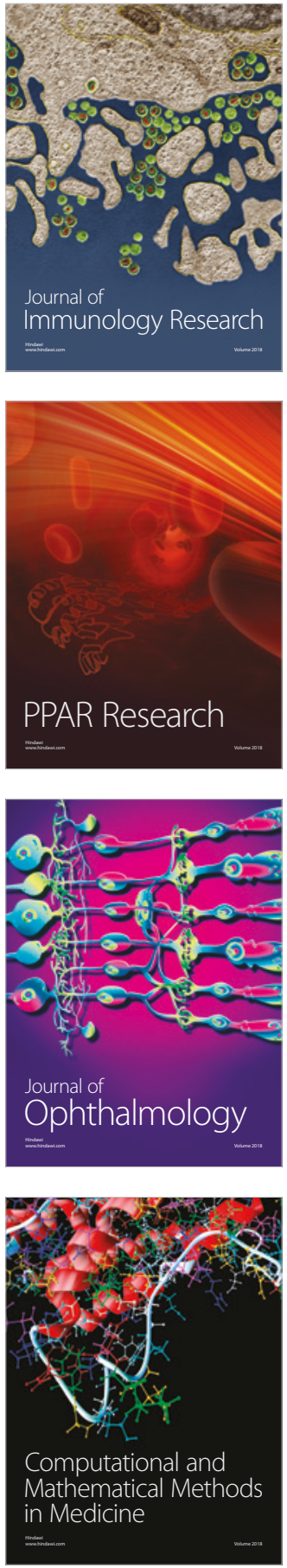

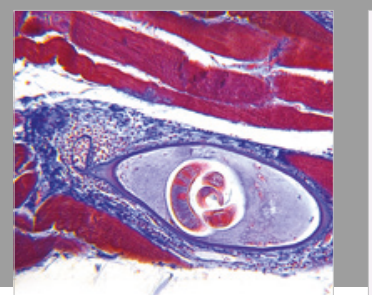

Gastroenterology Research and Practice

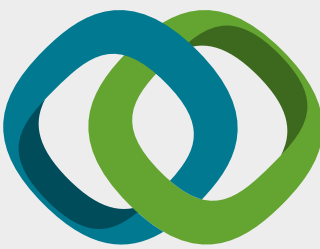

\section{Hindawi}

Submit your manuscripts at

www.hindawi.com
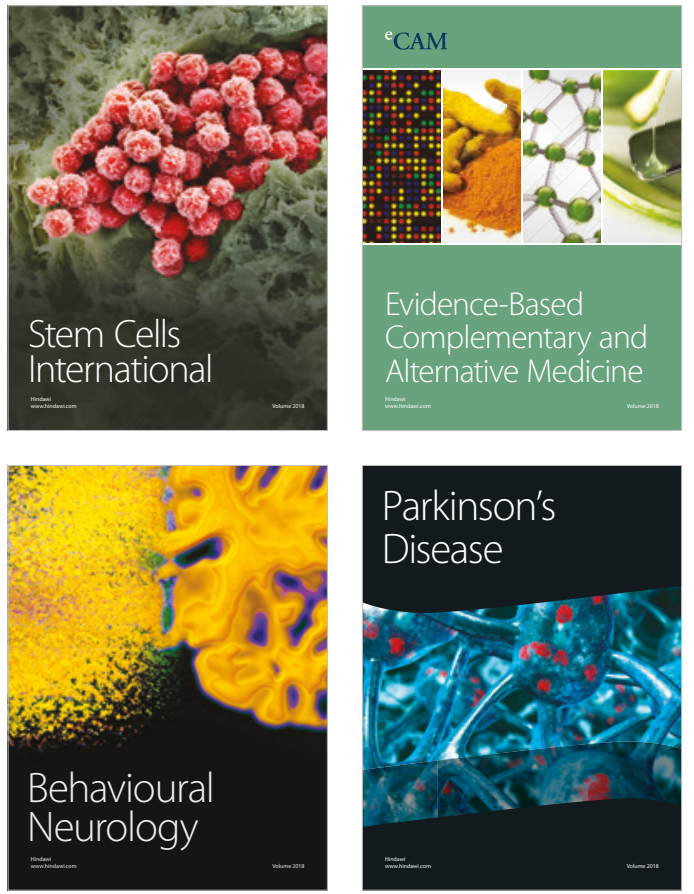

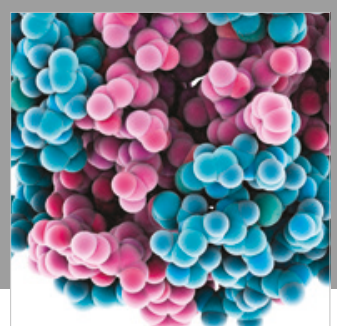

ournal of

Diabetes Research

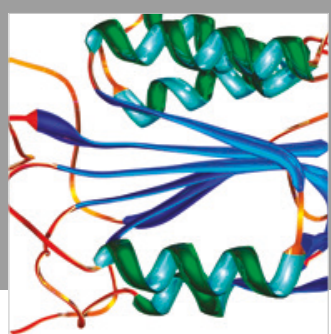

Disease Markers
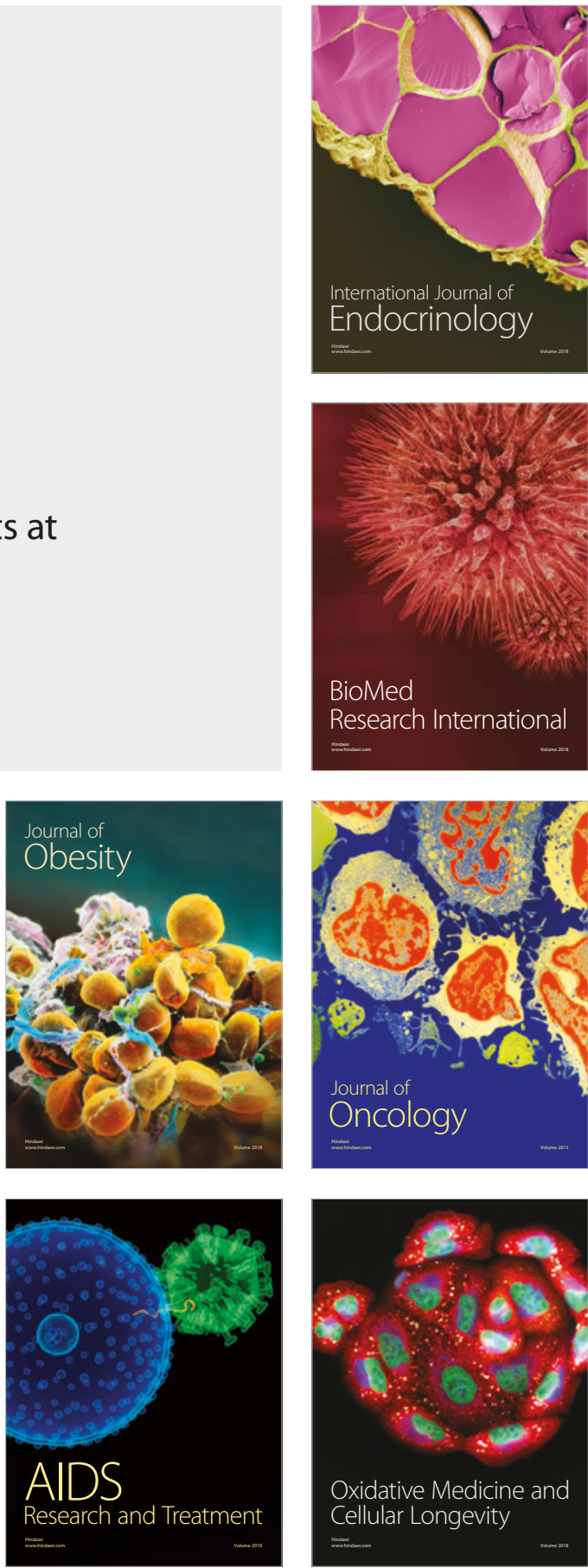